\title{
Tailoring the EB-PVD columnar microstructure to mitigate the infiltration of CMAS in 7YSZ thermal barrier coatings
}

\author{
R. Naraparaju*, M. Hüttermann, U. Schulz, P. Mechnich \\ German Aerospace center (DLR), Institute of Materials Research, Cologne 51170, Germany
}

\section{A R T I C L E I N F O}

\section{Article history:}

Received 14 April 2016

Received in revised form 12 July 2016

Accepted 21 July 2016

Available online $\mathrm{xxx}$

\section{Keywords:}

CMAS

Thermal barrier coatings

7YSZ

Infiltration

EB-PVD coatings

\begin{abstract}
A B S T R A C T
The CMAS associated degradation of 7YSZ TBC layers is one of the serious problems in the aero engines that operate in dusty environments. CMAS infiltrates into TBC at high temperatures and stiffens the TBC which ultimately loses its strain tolerance and gets delaminated. The EB-PVD technique is used to coat TBCs exhibiting a columnar microstructure on parts such as blades and on vanes. By varying the EB-PVD process parameters, columnar morphology and porosity of the 7YSZ coating is changed and its effect on the CMAS infiltration behaviour is studied in detail. Two different TBC pore geometries were created and infiltration experiments were carried out at $1250^{\circ} \mathrm{C}$ and $1225^{\circ} \mathrm{C}$ for different time intervals. The $7 Y S Z$ coating with more 'feathery' features has resulted in higher CMAS resistance by at least by a factor of 2 than its less 'feathery' counterpart. These results are explained on the basis of a proposed physical model.
\end{abstract} (C) 2016 Elsevier Ltd. All rights reserved.

\section{Introduction}

The durability and the functionality of $7 \mathrm{wt} . \%$ yttria stabilised zirconia thermal barrier coating (7YSZ TBC) in gas turbines is severely affected by the infiltration of molten deposits of calcium magnesium aluminosilicate (CMAS) [1-8]. CMAS infiltrates into the porous 7YSZ TBC (which protects the underneath Ni-based component against extreme thermal environment) and eventually solidifies upon flowing down the thermal gradient. This causes the stiffening of the TBC which ultimately loses its strain tolerance and gets delaminated. Electron beam physical vapour deposition (EBPVD) is used to coat TBCs on rotating parts such as blades. The TBCs that are coated with the above technique generally exhibit a columnar structure. Inter-columnar gaps are infiltrated with CMAS under the influence of capillary forces. The CMAS infiltration depth is governed by several factors such as viscosity, surface tension of the melt, operation temperature and shape of the inter-columnar gaps. The chemistry of the CMAS sand depends on geographical location and its viscous behaviour which is greatly influenced by the temperature $[9,10]$. Melting points of these CMAS variants fall in the range between $1135^{\circ} \mathrm{C}-1270^{\circ} \mathrm{C}$, however, partial melting may start even at lower temperatures. The temperature gradient in TBCs is an important factor in defining the infiltration depth as the temperature gradient influences both viscosity and the crystallisa-

\footnotetext{
* Corresponding author.

E-mail address: ravisankar.naraparaju@dlr.de (R. Naraparaju).
}

tion of the CMAS melt. Field specimens returning from service in CMAS-loaded areas reveal that the penetration of CMAS in EB-PVD coatings can reach up to the half of the TBC thickness, which is still capable of inducing delamination [11].

Several efforts have been made not only to understand the mechanisms of CMAS attack on TBCs but also to develop novel TBCs that are CMAS resistant. Gd-zirconate coatings have been tested as an alternative to 7YSZ coatings as the former promises a scope for mitigation of CMAS infiltration. Gd-zirconate reacts quickly with CMAS and forms a crystalline by-product of calcium oxy-apatite and seals the capillary channels against further CMAS penetration $[12,13]$. However, its reliability against low calcia containing CMAS and volcanic ash compositions is somewhat questionable due to insufficient calcium amounts necessary to stabilise the apatite phase [14]. Another promising strategy is to modify the 7YSZ composition and force CMAS to crystallise and stop its infiltration. $20 \mathrm{~mol} \%$ of $\mathrm{Al}_{2} \mathrm{O}_{3}$ and $5 \mathrm{~mol} \%$ of $\mathrm{TiO}_{2}$ in the form of a solid solution were incorporated into 7YSZ by means of solution precursor plasma spray process (SPPS) [15]. But long time stability of this mitigation mechanism was not comprehensively proved. One of the effective coatings to mention is $51 \mathrm{wt} . \% \mathrm{Y}_{2} \mathrm{O}_{3}$. $49 \mathrm{wt} . \% \mathrm{ZrO}_{2}$ coated with MOCVD technique [16]. This coating has formed crystalline products such as oxyapatite, mellilite and garnets with all available cations as reaction product with CMAS and showed best infiltration resistance.

It is known from the literature that the thermal conductivity of a TBC coating depends on its porosity, mainly along horizontal splat boundaries in APS coatings and on the porosity between 
Table 1

EB-PVD process parameters applied in this study and associated morphology.

\begin{tabular}{lll}
\hline Morphology & Substrate temperature & Rotation speed (rpm) \\
\hline 'normal' & $1000^{\circ} \mathrm{C}$ & 12 \\
'feathery' & $840^{\circ} \mathrm{C}$ & 30 \\
\hline
\end{tabular}

the feather-arm features in EB-PVD TBCs. Previous own work has demonstrated that the variation of EB-PVD process parameters alters the resulting columnar morphology and porosity of the coating. Thermal conductivity was greatly affected by these morphological alterations which mainly have changed the amount, size and shape distribution of pores and secondarily the pore surface area available at the cross section perpendicular to the heat flux. To the best knowledge of the authors the influence of TBC microstructure on CMAS infiltration is rarely reported in the literature, neither for APS nor for EB-PVD coatings.

In this paper, columnar morphology and density of EB-PVD 7YSZ columns is changed and its effect on the CMAS infiltration behaviour is studied in detail.

\section{Experimental procedure}

\subsection{Processing and materials}

EB-PVD coatings were produced in-house at DLR with a coater that has a maximum electron beam power of $150 \mathrm{~kW}$. Evaporation was carried out from a single ingot having the standard $7 \mathrm{wt} . \% \mathrm{Y}_{2} \mathrm{O}_{3}$ stabilised $\mathrm{ZrO}_{2}$ composition. Vapour phase is deposited on plane alumina substrates under conventional rotating mode as described in Ref. [17] (i.e. by mounting the substrates on a holder with its axis perpendicular to the evaporation source). $400-440 \mu \mathrm{m}$ thick 7YSZ coatings were deposited on $1 \mathrm{~mm}$ thick alumina substrates. This abnormal coating thickness was taken in order to carry out longer CMAS infiltration tests. The substrates were rotated at two different speeds and two different sample temperatures were targeted to get two different coating morphologies denoted as 'normal' and 'feathery'. Table 1 lists the obtained morphologies and their respective coating parameters. These parameters were selected on the basis of previous studies in house which have shown considerable difference in the porosity and configuration of the coating $[17,18]$.

\subsection{Synthesis and deposition of CMAS}

The CMAS composition was derived from real engine deposits (22 $\mathrm{CaO}, 8 \mathrm{MgO}, 18 \mathrm{Al}_{2} \mathrm{O}_{3}, 40 \mathrm{SiO}_{2}, 10 \mathrm{FeO}$ and $2 \mathrm{TiO}_{2}$ all in wt.\%) as used by the authors previously [6]. The melting interval of this composition lies in the range of $1230-1250^{\circ} \mathrm{C}$. CMAS was synthesised by means of co-decomposition of Me-nitrates $(\mathrm{Me}=\mathrm{Al}, \mathrm{Ca}$, $\mathrm{Mg}, \mathrm{Fe}$ ) together with $\mathrm{SiO}_{2}$ and $\mathrm{TiO}_{2}$ powders (Merck, Darmstadt, Germany), followed by a heat treatment at $1250^{\circ} \mathrm{C}$ for one hour. CMAS has been completely molten after this heat treatment and $\mathrm{X}$-ray diffraction results on the powder after cooling/at room temperature showed that it is mainly crystallised in to pyroxene and anorthite phases. The CMAS powder was deposited as a paste on top of EB-PVD 7YSZ TBCs in the amounts of $20 \mathrm{mg} / \mathrm{cm}^{2}$. Alumina substrates were used in this study to avoid heavy damage of metallic substrates and bond coats at the high infiltration temperatures.

\subsection{Infiltration and characterisation}

Infiltration experiments were conducted at temperatures of 1225 and $1250^{\circ} \mathrm{C}$ in a chamber furnace. The duration of the infiltration was varied in between $10-50 \mathrm{~h}$. Standard metallographic techniques were used in preparing cross-sections of samples in various conditions. SEM (DSM Ultra 55, Carl Zeiss NTS, Wetzlar,
Germany) equipped with an EDS system (Inca, Oxford Instruments, Abingdon, UK) was used to study the reacted zones and the infiltration depths. CMAS infiltration depths are measured with the help of high magnification imaging and confirmed by EDS elemental mapping. With the help of optical microscope and the Axio vision software the in-plane porosity close to the surface was estimated in both cases.

\section{Results}

\subsection{Microstructural differences between 'normal' and 'feathery' morphology}

To compare the morphological differences between 'normal' and 'feathery' structures, SEM top views are presented in Fig. 1(a) $\&$ (b) respectively. These morphological differences in their column diameter and size were produced by varying the EB-PVD process parameters given in the previous section and Table 1. Variation in substrate temperature and rotation speed is one of the influencing parameter which defines the growing process of the columns. Specifically the interdependences between the shadowing effect of the columns tips and the diffusion process in the growing surfaces are altered by such modifications. In order to reveal the intercolumnar gap structure in more detail, the coatings were polished from top to few microns down and the corresponding micrographs are presented in Fig. 1(c) \& (d).

The major difference between the 'normal' and the 'feathery' morphology is the reduction in the diameter of the individual columns. Measurements of the dimensions revealed that the individual diameter of the columns lies in the range of $15-30 \mu \mathrm{m}$ for the 'normal' structure and $12-17 \mu \mathrm{m}$ for the 'feathery' structure, respectively, i.e. the individual columns in 'feathery' structure are almost 50\% finer than in 'normal' structure. In consequence, the number of open channels in 'feathery' structure is increased compared to the 'normal' structure. This can be noticed from Fig. 1(c) and (d). The in-plane porosity close to the surface of the TBC in 'feathery' (12\%) is found to be 3 times higher than in 'normal' (4\%) structure. Even though these porosity measurements are based on the contrast ratio of the pores to the columns and the absolute values might not fully reflect the real porosity, they make a qualitative statement on the difference in inter-columnar porosity. Note that other pores that contribute to the overall porosity within EB-PVD TBCs (that is typically in the order of $25 \%$ ) such as intra-columnar fine pores and voids between feather arms have not been measured here.

The SEM cross-sectional micrographs of both variants are presented in Fig. 2. In order to understand the variation of columnar diameter, gap width, and length of the feather arms throughout the coating, measurements were done at each $50 \mu \mathrm{m}$ distance from top of the coating to the bottom. These measurements were repeated at 2 more places on the coating and the values are averaged over all 3 measured values (Fig. 3). The width and the shape of the columnar gaps in case of 'normal' structure remained almost uniform throughout the coating thickness whereas in case of 'feathery' structure a large variation in the shape and size can be noticed. In case of 'normal' morphology which was produced by employing higher substrate temperatures with slower rotation speed, a symmetrical arrangement of larger column diameters down up to $300-350 \mu \mathrm{m}$ is observed. The length of the feather-arm features surrounding the columns lies in the range of $1 / 5$ to $1 / 7$ th of the primary column cross section. Coatings with 'feathery' morphology exhibit a continuous drop in their columnar diameter from top to bottom (from $\sim 12$ to $2 \mu \mathrm{m}$ ) and the feather arm features occupy almost $1 / 2$ to $1 / 3$ of the primary column cross section. Moreover, the inter-columnar gaps in the 'feathery' structure are getting nar- 


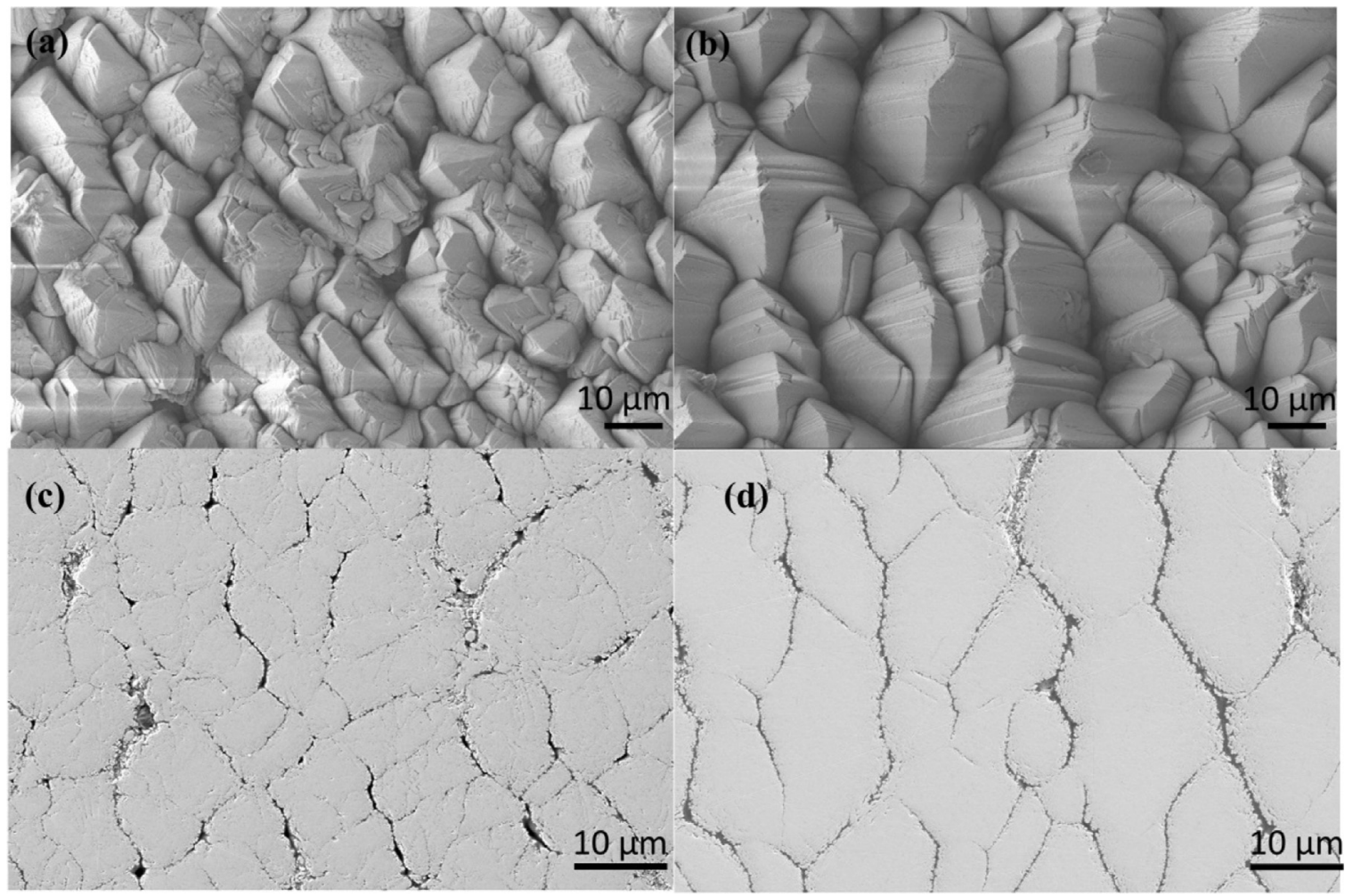

Fig. 1. SEM micrographs of as coated (a)\&(c) 'feathery', (b)\&(d) ‘normal' structures of EB-PVD 7YSZ coating. Top view (a) \& (b) and polished top view (c) \& (d).

rower at the lower part of the coating (from $200 \mu \mathrm{m}$ downwards) and the inter-columnar gaps are tilted and intersecting, i.e. the tortuosity of the gaps is higher compared to the 'normal' structure. These kinds of structures were initially created to achieve a low thermal conductivity. The thermal conductivity values for 'normal' and 'feathery' morphologies at room temperatures were found in previous studies to be 1.75 and $1.15 \mathrm{~W} / \mathrm{mK}$, respectively. The drop in the thermal conductivity of 'feathery' structures can be related to the higher porosity compared to 'normal' structure [18].

\subsection{Infiltration experiments}

\subsubsection{CMAS infiltration experiments at $1250^{\circ} \mathrm{C}$}

CMAS powders were deposited on the 'normal' and 'feathery' structured EB-PVD 7YSZ samples and heated isothermally at $1250^{\circ} \mathrm{C}$ for $10 \mathrm{~h}$, respectively. In both cases CMAS has infiltrated completely through the coating and reached the alumina substrate. i.e. $100 \%$ infiltration has happened. Fig. 4(b) shows the coating/substrate interface of the infiltrated 7YSZ coating. The individual columns have also reacted with the CMAS and most of the characteristic 'feathery' structure is lost and all intra columnar pores are filled with CMAS as shown in Fig. 4(a). YSZ has significantly dissolved and reacted to form a phase appearing as globular particles. EDS analysis (which is not shown here) of this globular phase revealed a lower yttria content than in the original 7YSZ and incorporation of $\mathrm{CaO}$ in solid solution. XRD analysis not presented here proves transformation of $\mathrm{t}-\mathrm{ZrO}_{2}$ to $\mathrm{m}-\mathrm{ZrO}_{2}$ that appears to be taken place during dissolution and re-precipitation.

To verify the structural effects, $1225^{\circ} \mathrm{C}$, a temperature where the CMAS is not completely molten (with higher viscosity [19]) has been chosen and infiltration experiments were carried out.

\subsubsection{CMAS infiltration experiments at $1225^{\circ} \mathrm{C}$}

Cross-sections of both 'normal' and 'feathery' after infiltrating the samples isothermally with CMAS for $10 \mathrm{~h}$ at $1225^{\circ} \mathrm{C}$ are pre- sented in Fig. 5. The microstructural features of CMAS reacted TBCs at $1225^{\circ} \mathrm{C}$ seem to be slightly different to that at $1250^{\circ} \mathrm{C}$. In the "normal" TBC, the columnar gaps are filled with CMAS but the intracolumnar pores seem to be less affected as in the case of $1250^{\circ} \mathrm{C}$. The feather arm structure is also lost in the upper part of the TBC but the CMAS has not penetrated down to the bottom as shown in Fig. 5(d). In the 'feathery' TBC, the feather arm structure can still be identified from $20 \mu \mathrm{m}$ depth onwards. The inter-columnar gaps are filled with the CMAS, but the infiltration did not proceed until the bottom of the coating as shown in Fig. 5(c). EDS spot analysis was done along the complete depth of the coating and infiltration depths are measured as explained in the experimental section. Infiltration depth values showed a significant difference between 'normal' and 'feathery' structures and measured as 10 and $20 \%$ of their total thickness, respectively.

Even though neither of the TBCs was completely infiltrated after $10 \mathrm{~h}$, a considerable effect of morphology on infiltration depth (in both 'normal' and 'feathery' structures) is observed. In order to verify this effect, time of infiltration tests was prolonged to 20 and $30 \mathrm{~h}$. After $20 \mathrm{~h}$ of isothermal heat treatment we found an even more clear difference in the infiltration depths. Whereas the 'normal' TBC was $100 \%$ infiltrated, the 'feathery' TBC was only infiltrated to half thickness. The corresponding SEM micrographs are presented in Fig. 6 . After $20 \mathrm{~h}$ at $1225^{\circ} \mathrm{C}$, the feather-arms have disappeared in the upper part of the TBC in both cases and the intra columnar porosity is filled with CMAS. Most of the inter-columnar gaps are filled with CMAS in case of the 'normal' structure and very large gaps are mostly visible with little CMAS as shown in Fig. 6(a) \& (b). However, the scenario at the bottom part of the coating (TBC/substrate) is quite different. In case of 'normal' TBC morphology CMAS has reached the substrate and the accumulated excess CMAS has reacted with alumina as shown in Fig. 6(c). In contrast, the 'feathery' TBC shows no CMAS at the substrate interface i.e. CMAS has not penetrated completely. It is not fully infiltrated by CMAS even after $30 \mathrm{~h}$ (Fig. 7(a)). However some CMAS was found 


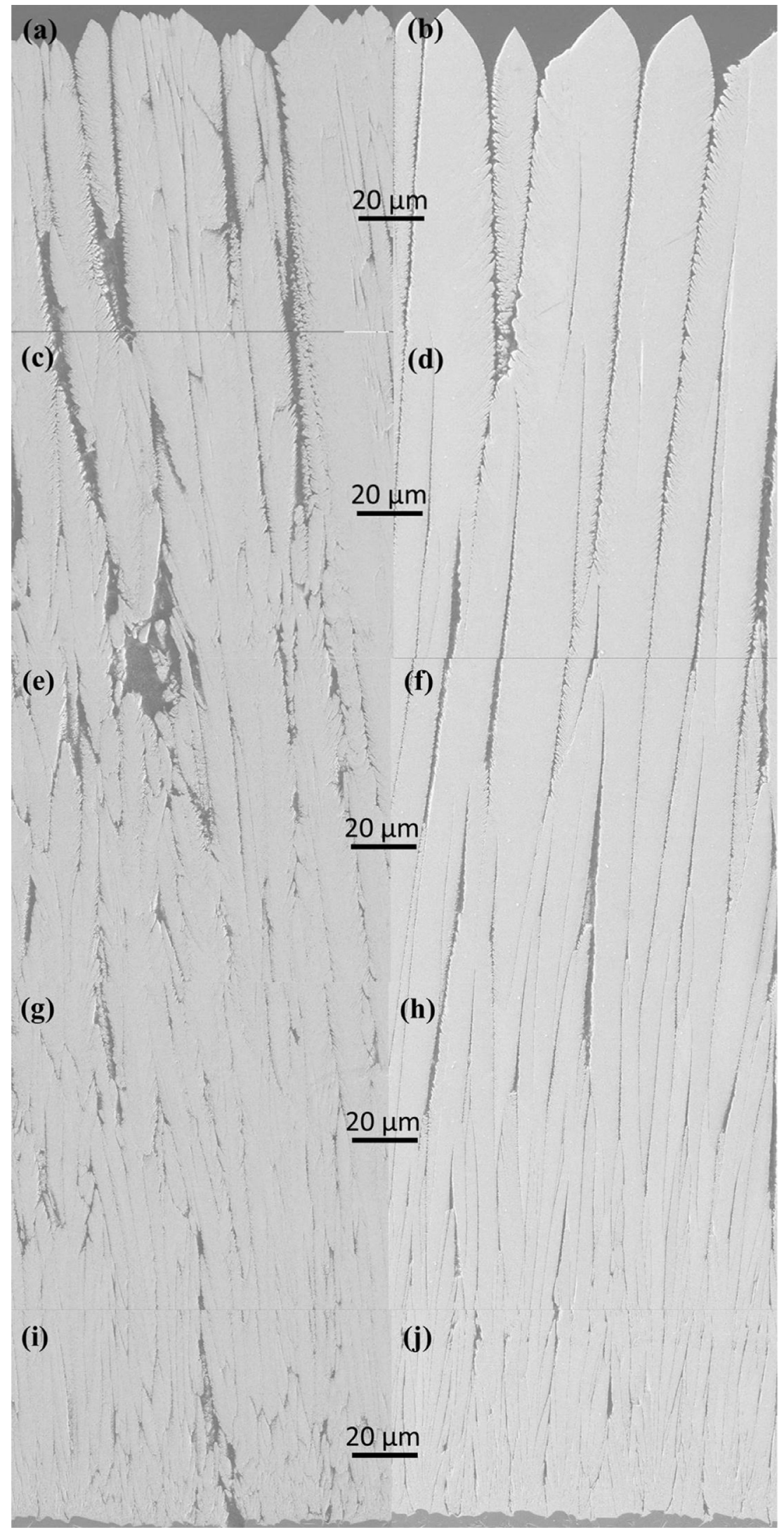

Fig. 2. SEM cross sectional images of as coated 'feathery' (a), (c), (e), (g) and (i), 'normal' (b), (d), (f), (h) and (j) EB-PVD 7YSZ coating. Distance from top (a)\&(b) 0-100 $\mu$ m, (c)\&(d) $100-200 \mu \mathrm{m}$, (e)\&(f) $200-300 \mu \mathrm{m}$, (g)\&(h) 300-400 $\mu \mathrm{m}$ and (i)\&(j) $\sim 400-440 \mu \mathrm{m}$. 
(a)

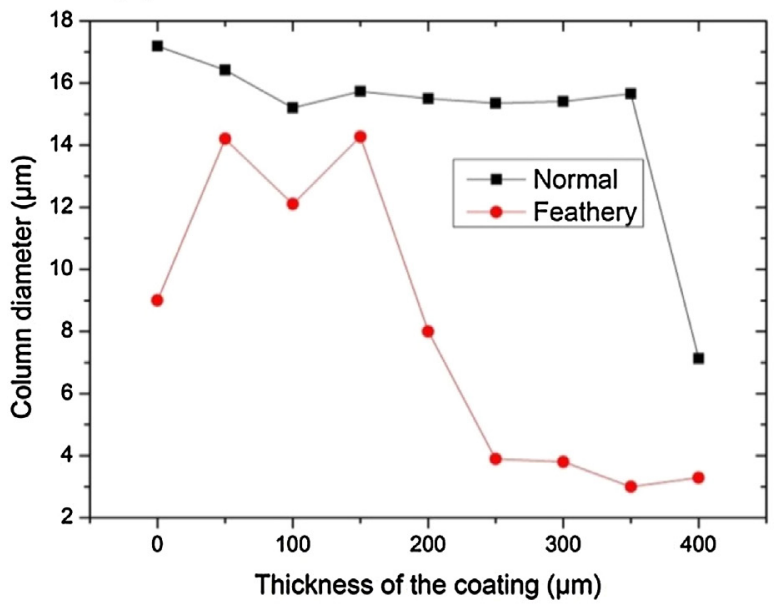

(b)

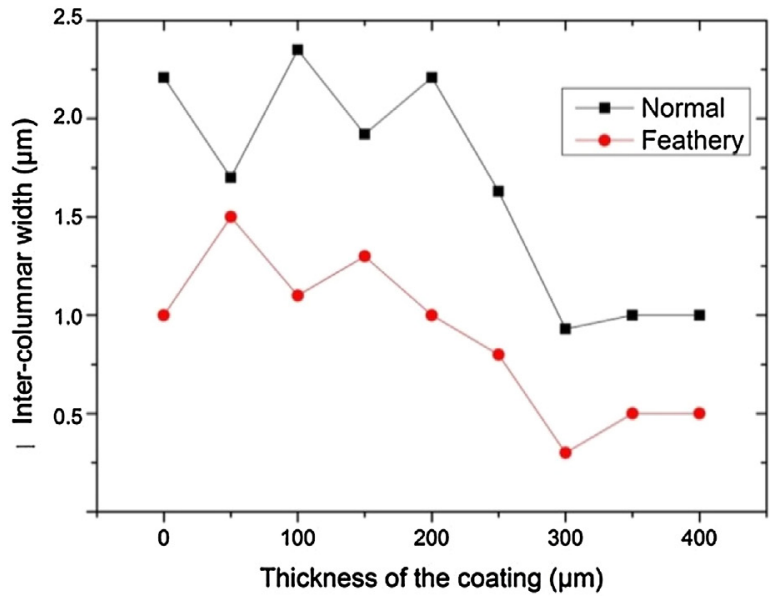

Fig. 3. EB-PVD columnar structure characteristics of 'normal' vs 'feathery' coating (a) columnar diameter and (b) inter-columnar gap width.

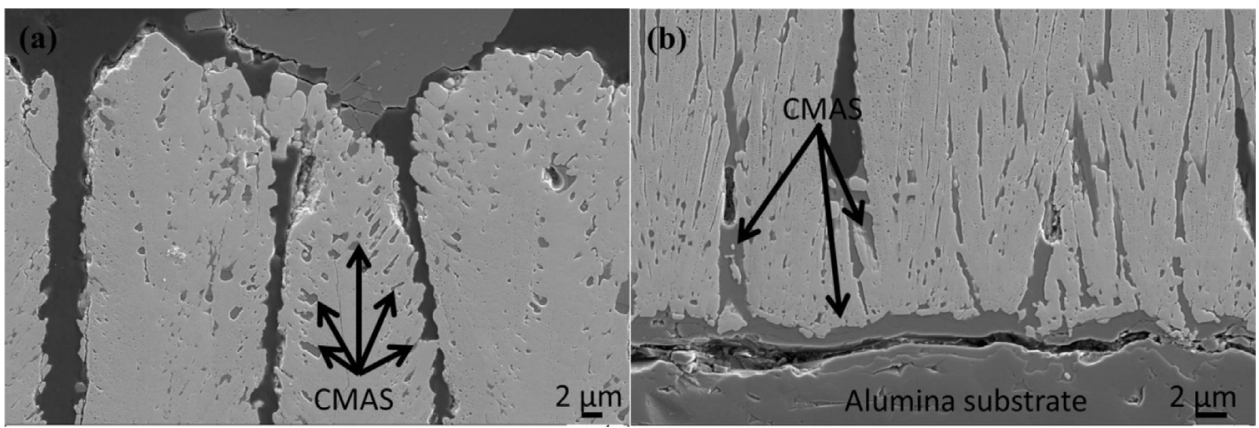

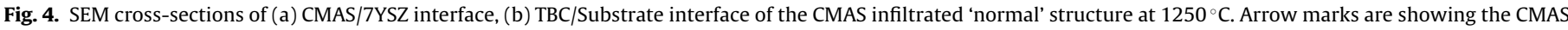
infiltration in the EB-PVD porosity.

in between the intra-columnar pores (confirmed by the Ca and $\mathrm{Si}$ elements in the EDS spot analysis) near the substrate as shown in Fig. 7(b). Infiltration depth in this case is drawn as $90 \%$ in Fig. 8 . The infiltration depths versus annealing times are summarized in Fig. 8.

\section{Discussion}

The modification in the EB-PVD columns in terms of intercolumnar and intra-columnar porosity was achieved by varying the deposition parameters. Based on the measurements given in Fig. 3 (column diameter and columnar gap width) and the observations of the morphology (representative examples are given in Figs. 1 and 2) a schematic illustration of both 'normal' and "feathery" structures is given in Fig. 9. The inclined lines illustrate schematically the feather-arms occurring at the outer column rims while the different width of the inter-columnar gaps is clearly visible.

When molten CMAS is deposited on such kind of EB-PVD columns a rapid infiltration happens through the porosity under the influence of capillarity forces. Basically there are two pore channels available for the infiltration. One is the inter-columnar gap which is aligned parallel to the principal flow direction. The second channel is the gap between two feather-arms inclined to the principle flow direction. These gaps can also be considered as capillary tubes with certain length allowing the molten CMAS to infiltrate through them. As a result, the amount of total CMAS flow in vertical direction in the TBC will be also distributed in to the 'feather-arm' channels. The inter-columnar gap width of the 'normal' TBC morphology is almost twice of that of the 'feathery' structure as shown in Figs. 3 and 8. If an inter-columnar gap is assumed as a hollow tube of radius ' $r$ ' with a height ' $h$ ', Darcy's law for the steady state uni-directional flow of a viscous liquid with a viscosity $\mu$ can be written as [20]

$\frac{\partial p}{\partial x}=-\frac{\mu v}{k} \cong \Delta p=-\frac{\mu}{k} \frac{x \partial x}{\partial t}$

where $k$ is the permeability of the medium, $v$ is the velocity of the liquid, $p$ is the pressure in the tube, $x$ is the infiltrated distance and $t$ is the time taken for the infiltration. If the capillary pressure is considered as the driving force for the infiltration, $\Delta p$ can be written as

$\Delta p=\frac{\sigma \cos \theta}{r}$

where $\sigma$ is the surface tension and the $\theta$ is the contact angle between the liquid CMAS and the surface. Complete wetting occurs when the contact angle is $0^{\circ}$ as the droplet turns into a flat puddle (i.e. $\theta=0$ ). By inserting Eq. (2) into (1) and integrating over the whole length of the tube, the time required by the liquid to infiltrate a depth of ' $h$ ' is derived which is shown in Eq. (3).

$t=\frac{\mu r h^{2}}{2 \sigma k}$

The permeability $k$ of the medium is an important factor which defines the speed of the infiltration. It depends on various factors such as overall porosity, pore connectivity, and shape factor of the pores. A well-known relationship correlating permeability with properties of pores is developed by Kozeny and later modified 


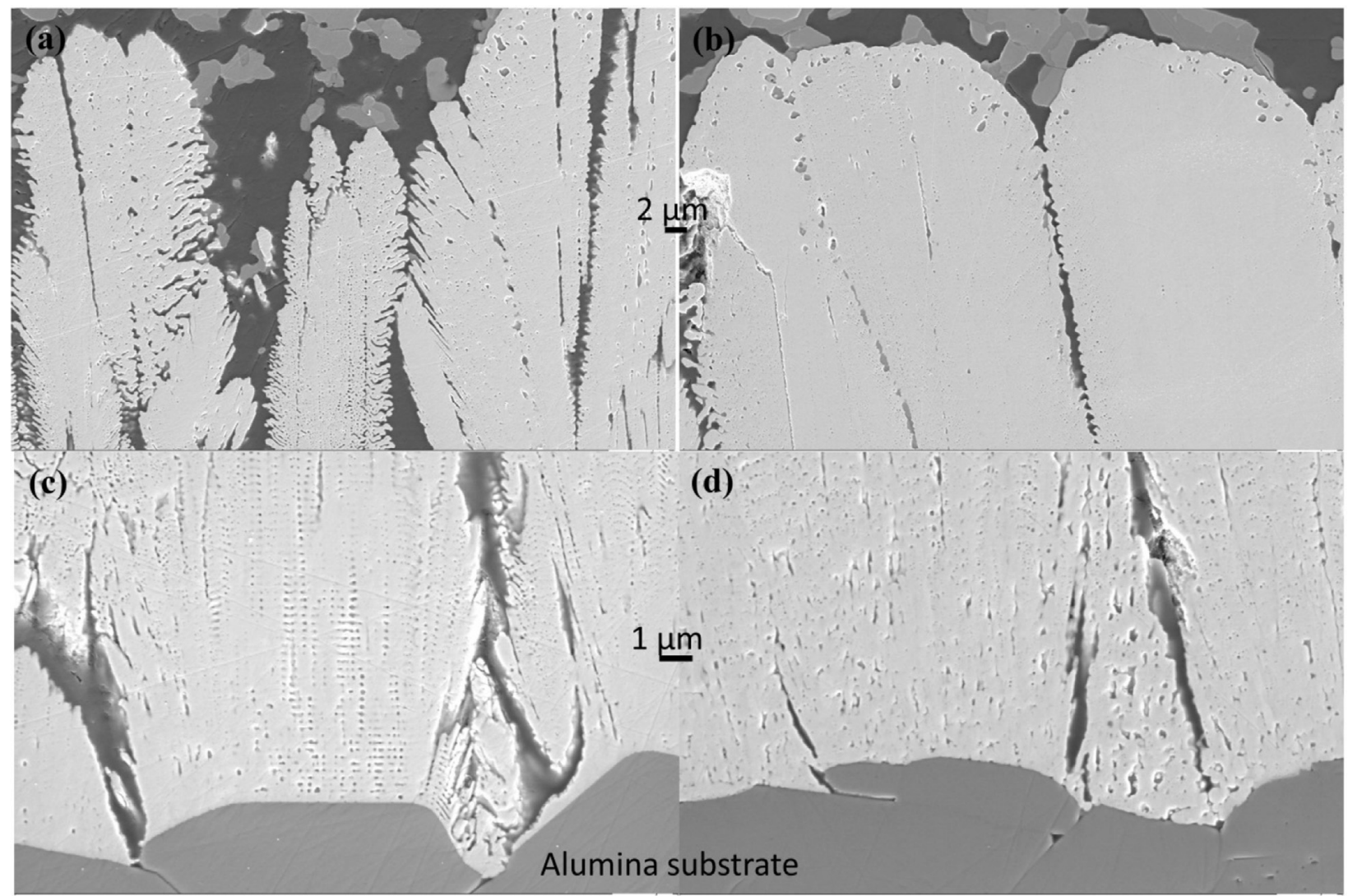

Fig. 5. SEM cross sections of CMAS infiltrated 7YSZ at $1225^{\circ} \mathrm{C}$ after $10 \mathrm{~h}$. (a) 'feathery' (top), (b) 'normal' (top), (c) 'feathery' (bottom) and (d) 'normal' bottom (bottom).

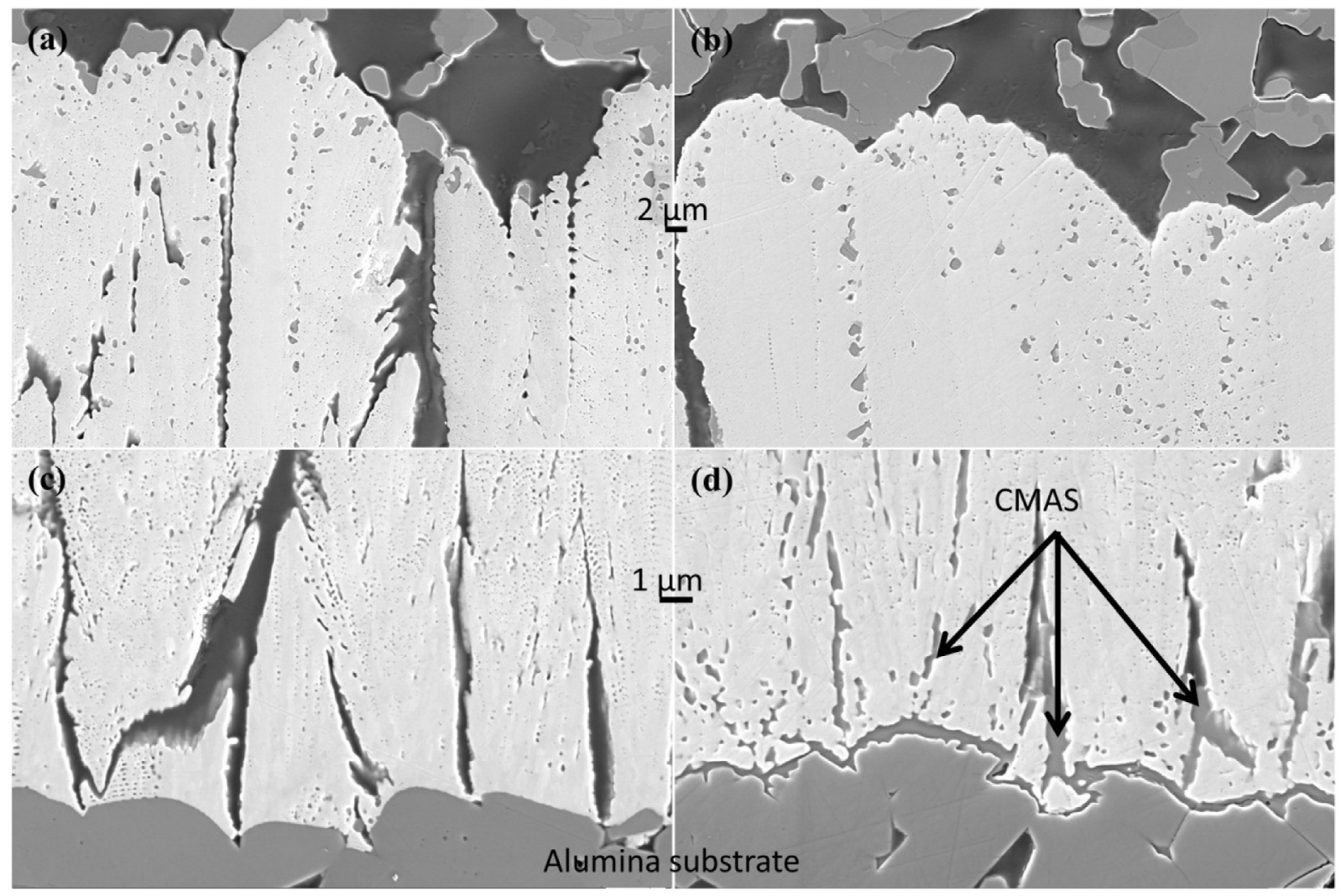

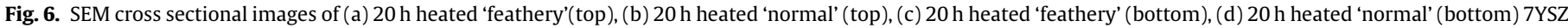
coating. 


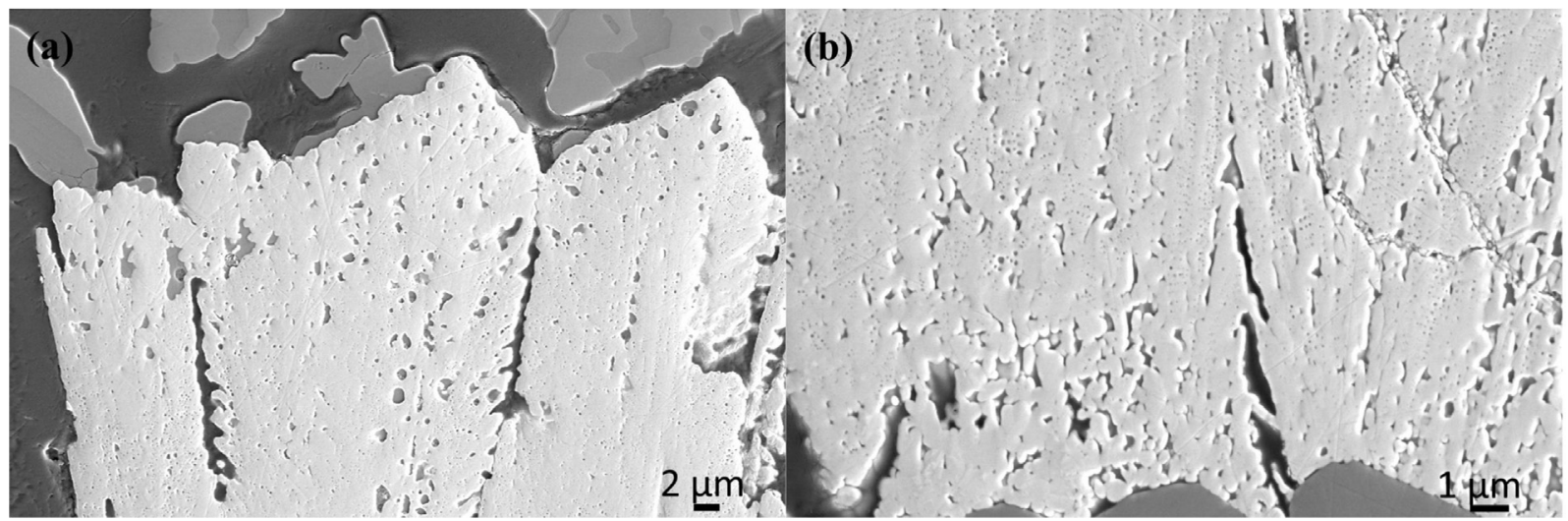

Fig. 7. SEM cross sectional images of 'feathery' (a) $30 \mathrm{~h}$ heated (top), (b) $30 \mathrm{~h}$ heated (bottom) 7YSZ coating.

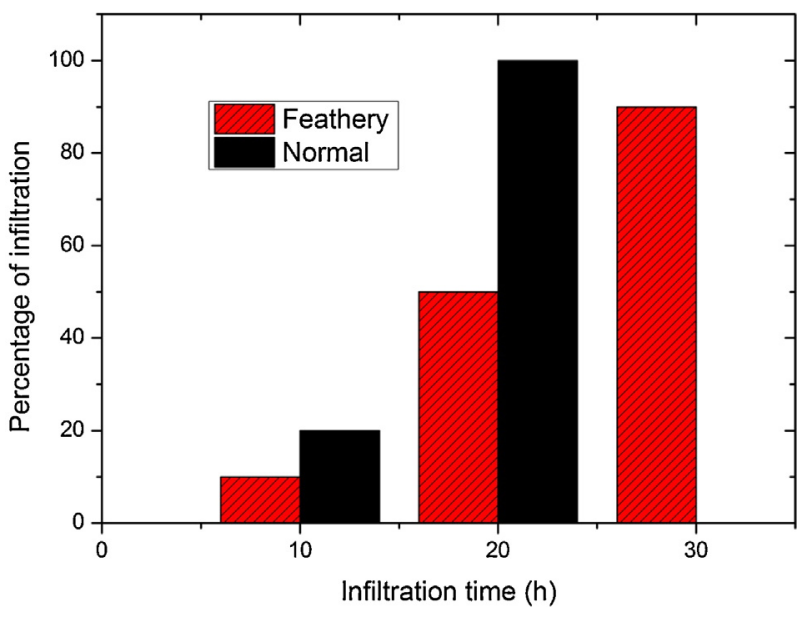

Fig. 8. Infiltration depth versus time of infiltration at $1225^{\circ} \mathrm{C}$ for both 'normal' and 'feathery' 7YSZ structures.

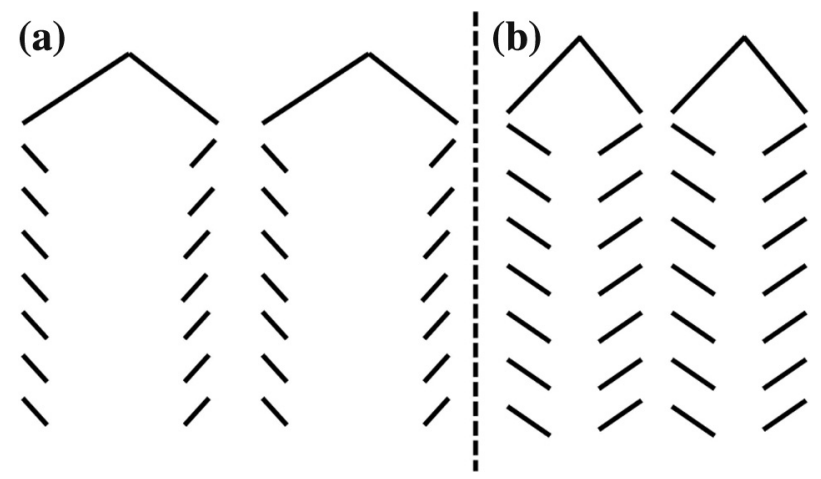

Fig. 9. Schematic representation of the (a) 'normal' and (b) 'feathery' columnar features.

by Carman and is given by Eq. (4) for an open pipe model (capillary tube) $[21,22]$.

$k=\frac{r^{2} \varphi^{2}}{8 \tau(1-\varphi)^{2}}$

$\varphi$ is the overall pore fraction of the medium and $\tau$ is the tortuosity of the medium. The geometrical definition of tortuosity is given as 'the reciprocal of the average ratio between the straight distance connecting two points and the actual path length inside the porous medium' [23]. Although this definition is widely used in the literature, it is not universally accepted. The tortuosity of a porous media in general depends on the pore volume fraction, shape, and the connectivity. Even though Carman experimentally found that a value of $\sqrt{ } 2$ should be chosen for tortuosity [24], it is also shown in other work that tortuosity can range from 1 to 4 in beds packed with non-uniform spheres depending on factors such as packing, arrangement, media homogeneity and channel shape [25-27]. In one of the simulation studies it was found that the tortuosity decreases with the increase in porosity in a parabolic manner and in the low porosity region this change is higher [23]. Inserting Eq. (4) into (3) gives the final Eq. (5) to calculate the time of infiltration.

$t=\frac{4 \mu h^{2} \tau}{r \sigma} \frac{(1-\varphi)^{2}}{\varphi^{2}}$

i.e. for a given temperature, viscosity and height of the channel the infiltration time is directly proportional to the tortuosity and inversely proportional to the radius of the capillary tube. Eq. (5) predicts that if the overall porosity increases then the time for infiltration should decrease. In this present study, the 'feathery' TBC exhibited a higher amount of porosity derived from the inter-columnar gaps, lower inter columnar gap widths and higher tortuosity (see Fig. 3(a) and (b)) than the 'normal' TBC. The higher 'feather-arm' porosity has contributed heavily to the increase in the overall porosity of 'feathery' structure. According to Eq. (5) the higher porosity of 'feathery' would result in a shorter infiltration time - opposite to the present findings. However, the gaps in between the deeper 'feathery' arms would not add to the main infiltration direction; they rather distribute the flow of CMAS and decrease the infiltration depth along the main TBC thickness as shown schematically in Fig. 10 i.e. in addition to the porosity it is also very important to know how all the pores are inter-connected. The higher length of the feathery gaps and lower inter columnar gap width in case of 'feathery' structure in comparison to 'normal' structure would result in a reduced infiltration depth. Table 2 summarizes the overall microstructural modifications applied on the 7YSZ system and the corresponding change in the infiltration depth of the CMAS.

At $1250^{\circ} \mathrm{C}$, there was no difference in the infiltration behaviour in both TBC morphologies, i.e. both have been $100 \%$ infiltrated. This infiltration behaviour is expected at this temperature because CMAS is in complete molten state with low viscosity (melting point $\sim 1250^{\circ} \mathrm{C}$ ) [19]. The viscosity of the CMAS composition was estimated at $1250^{\circ} \mathrm{C}$ and $1225^{\circ} \mathrm{C}$ as -0.25 and $-0.10(\log \eta$ (Pa.s) respectively by using the Giordano model for silicate melts. Such low viscosity liquids are readily flowing on the TBC surface, the infiltration is faster and structural effects such as porosity and shape of the columns have very little influence on the infiltration kinetics. At $1225^{\circ} \mathrm{C}$, a clear difference in the infiltration behaviour was 
Table 2

Summary of the microstructural changes and corresponding influence on the CMAS infiltration depth obtained in this study.

\begin{tabular}{|c|c|c|c|c|c|}
\hline TBC Morphology & $\begin{array}{l}\text { In-plane } \\
\text { Porosity(\%) }\end{array}$ & $\begin{array}{l}\text { Column } \\
\text { Diameter }(\mu \mathrm{m})\end{array}$ & $\begin{array}{l}\text { Gap width/diameter } \\
(\mu \mathrm{m})\end{array}$ & Tortuosity & $\begin{array}{l}\text { Infiltration at } 1225^{\circ} \mathrm{C} \\
\text { after } 10 \mathrm{~h}(\%)\end{array}$ \\
\hline Normal & 4 & $17-20$ & $1.7-2.3$ & lower & $100 \%$ \\
\hline Feathery & 12 & $8-15$ & $1-1.2$ & higher & $50 \%$ \\
\hline
\end{tabular}

\section{Mechanism: Splitting the path}
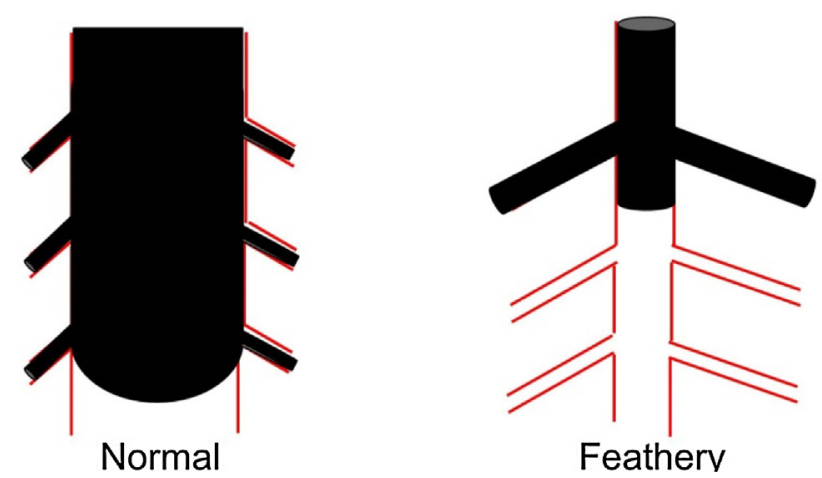

Fig. 10. Schematic representation of inter-columnar gaps and inter-feathery gaps as small capillary tubes used by CMAS as infiltration channels.

observed after $10 \mathrm{~h}$. The infiltration depth in 'feathery' TBC (10\%) was found to be half of that of the 'normal' TBC. During infiltration tests at $1225^{\circ} \mathrm{C}$ CMAS melt contains still some unmolten crystals and hence may have a higher viscosity. After $20 \mathrm{~h}$, the difference in the infiltration depth remained similar as the 'feathery' TBC was infiltrated up to $50 \%$ whereas the 'normal' TBC was infiltrated fully. Even after $30 \mathrm{~h}$ the 'feathery' TBC was infiltrated only to $90 \%$. The CMAS infiltration kinetics was reduced by almost $50 \%$ by increasing the tortuosity, decreasing the columnar gap width and increasing the porosity. These alterations and their effects on the infiltration time were in good agreement with the model proposed above and with the literature. However, the effects seen in this study hold well only in the high viscosity regime of the melt or at lower viscous regimes. Very short time infiltration tests are needed to confirm the microstructure effect and these experiments are carried out presently. In real TBC operating under a thermal gradient, these effects can play an important role in changing the infiltration depth and hence the life time of the TBC layer. Under temperature gradients in an internally cooled blade or vane, at a specific height within the TBC it is very likely that a temperature regime occurs where the above mentioned influence of morphology on infiltration depths is operating. Hence a TBC having features of the feathery microstructure or other changes in morphology that reduce the driving forces for infiltration in accordance with Eq. (5) is favourable. It is assumed that such a TBC will suffer to a lower extent from infiltration, TBC stiffening, and ultimately TBC spallation.

The Carman-Kozeny model which was described above is valid for the most simplified open pipe model. Applying this to the intercolumnar gaps is a quite large simplification. However, in reality EBPVD columns appear rather like a pipe with an inside kernel (Fig. 10) as can be seen from the original microstructure of EB-PVD porosity as shown in Fig. 1(c) and (d). The inter-columnar gaps surround the whole column, and hence can be more accurately described as such a kernel tube, knowing that the shape of a column is not ideal circular but elongated in two directions (one is the rotational axis and the other is perpendicular to it).

Dvorkin has extended the Carman-Kozeny model for the permeability of a pipe with a kernel (black circle in Fig. 11) and Eq. (6)

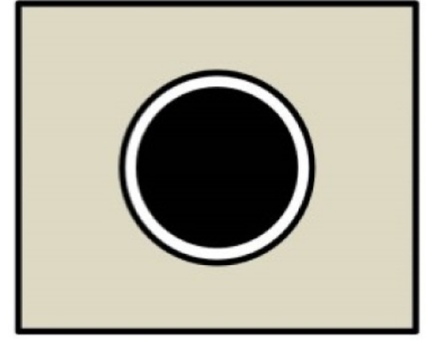

Fig. 11. Schematic representation of the pipe with a kernel inside.

represents the permeability $k$ in terms of pipe (white circle) outer radius 'b' and kernel radius 'a' [28].

$k=\frac{\phi}{8 \tau^{2}} b^{2}\left[1+\frac{a^{2}}{b^{2}}+\left(1-\frac{a^{2}}{b^{2}}\right) \cdot \frac{1}{\ln \left(\frac{a}{b}\right)}\right]$

The variation in the pipe and kernel radius causes a change in the permeability of the medium. To demonstrate the change in the permeability value from the open pipe model to the pipe with a kernel model few assumptions are made such as taking the overall pore fraction $(\phi)$ as constant $(50 \%)$ and a tortuosity factor $(\tau) 1$. Calculated permeability values using Eqs. (4) \& (6) are drawn graphically in Fig. 12 by changing the pipe radius given on the $\mathrm{X}$-axis. Two different cases are considered here in this calculation.

In the open pipe model (green triangles) the radius of the pipe is varied between $1 \mu \mathrm{m}$ and $5.5 \mu \mathrm{m}$. The second curve (blue rhombus) is a fixed kernel radius of $4 \mu \mathrm{m}$ and the pipe annulus is varied between 1 and $4 \mu \mathrm{m}$. i.e. the radius b of the pipe is varied between 5 and $9 \mu \mathrm{m}$ ( $4 \mu \mathrm{m}$ from 'a' $+1 \mu \mathrm{m}$ from 'b'). Consequently, to compare similar gap-widths, the permeability of the open pipe radius of $1 \mu \mathrm{m}$ must be compared to $5 \mu \mathrm{m}$ pipe radius of the kernel constant curve. The permeability given by the open pipe model $\left(0.125 \mathrm{~m}^{2}\right)$ is found to be three times that of the pipe with kernel model $\left(0.041 \mathrm{~m}^{2}\right)$ and the slope of the permeability curve in case of the open pipe model is quite higher than that of the pipe with the kernel model. That means as the pipe radius value increases the difference between the two model predictions is getting higher. Consequently, by not choosing the proper permeability model one can under/over estimate the CMAS infiltration depth in EB-PVD columns considerably.

Permeabilties of 'normal' $\left(k_{n}\right)$ and 'feathery' $\left(k_{f}\right)$ structures are calculated by using both open pipe and pipe with kernel model from the Eqs. (4) \& (6). For the open pipe model a radius $\mathrm{r}$ of $1 \mu \mathrm{m}$, (using a mean value for diameter) and a porosity value of $4 \%$ were considered for the normal structure, while $0.5 \mu \mathrm{m}$ radius and $12 \%$ was used for the feathery structures, as given by Table 2 . As the tortuosity factor $(\tau)$ is unknown, a value of 1 is considered for both 'normal' and 'feathery' structures in this calculation (in both models). The calculated ratio according to the open pipe model is given as

$\frac{k_{f}}{k_{n}}=\frac{1}{5.97}$

In the pipe with kernel model, $\mathrm{a}=8.5, \mathrm{~b}=9.5 \mu \mathrm{m}$ are taken for the 'normal' structure. For 'feathery' structure values of $\mathrm{a}=4.5, \mathrm{~b}=5 \mu \mathrm{m}$ 


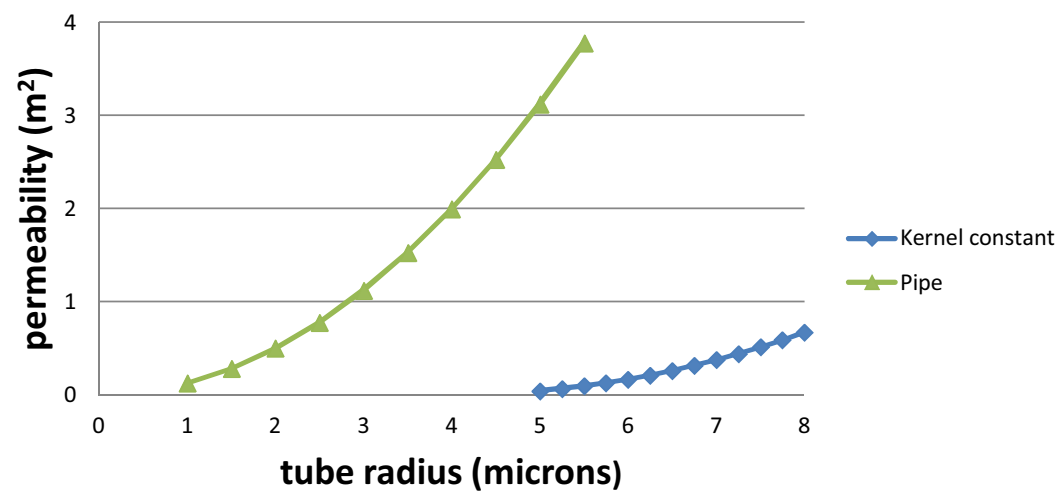

Fig. 12. Comparison of the permeability values using the open pipe and the pipe with a kernel model for a given porosity.

are considered, using the same porosities as for the open pipe calculation. The corresponding permeability ratio is calculated as

$\frac{k_{f}}{k_{n}}=\frac{1}{1.33}$

In both cases it is evident that the permeability of the 'feathery' structure is smaller than that of the 'normal' structure, however, the ratios differ each other by a factor of 4.5. According to Eq. (3) it is known that a lower permeability of a medium gives a longer infiltration time. The experimental results show that the infiltration depth of the 'feathery' structure is only $\frac{1}{2}$ than that of 'normal' structure for the same infiltration time, although the ratio is not fully accurate since the full infiltration of the normal structure may have been reached at shorter $\frac{1}{3}$ times than the presently evaluated $10 \mathrm{~h}$. This would shift the experimental ratio towards $\frac{1}{2.5}$ or $\frac{1}{3}$. As the permeability can be correlated to the infiltration depth, the factor of $\frac{1}{1.33}$ (pipe with $\frac{1}{5.97}$ kernel model) comes closer to this value rather than from the open pipe model $\frac{1}{5.97}$. With the given number of assumptions, this prediction is quite reasonable even though the overall porosity is different from the in-plane porosity and the tortuosity factor is unknown for both structures.

To validate these model predictions especially with the permeability and tortuosity elements, high temperature viscosity and contact angle values of the CMAS are in progress. In addition, systematic quick infiltration tests where exact infiltration depths for short intervals of time $\left(1-5 \mathrm{~min}\right.$ at $\left.1250^{\circ} \mathrm{C}\right)$ must be done. With all the experimentally obtained values, a direct and more precise comparison of the two models can be done. Most importantly heat treatments prior to the CMAS exposure on the 7YSZ samples are currently carried out to remove a lot of inter-feathery gaps. This will provide more insight into the effect of tortuosity and feather arm lengths on the infiltration depth?

\section{Conclusions}

Microstructural modifications in the EB-PVD columns of 7YSZ TBCs were achieved by varying the process parameters. CMAS infiltration studies were performed on two different coating morphologies at $1225^{\circ} \mathrm{C}$ and $1250^{\circ} \mathrm{C}$. The main focus of this study was to determine the effect of coating morphology on CMAS infiltration resistance and the following conclusions can be made from the results.

- CMAS infiltration results at $1250^{\circ} \mathrm{C}$ (low viscosity range) in a $100 \%$ infiltration for both morphologies after $10 \mathrm{~h}$. However, if controlled infiltration tests are carried out for shorter times an effect from microstructure can be observed.

- Infiltration tests at $1225^{\circ} \mathrm{C}$ (relative high viscosity range) for different time ranges demonstrate that the feathery structure show
CMAS infiltration at roughly half the depth of a normal EB-PVD structure. Increased tortuosity and the small variations in the morphological features of the columnar coating (higher length of the feather-arms and smaller inter-columnar gaps) play an important role here.

- The slowed down infiltration speed is anticipated to prolong the lifetime of EB-PVD TBCs under CMAS attack by a lower degree of infiltration, and hence by lower stresses in the TBC.

- The predictions from a simplified tube flow model demonstrate that the main mechanisms in the CMAS infiltration are governed by the viscosity of the fluid and the permeability of the medium (the latter being related to pore volume fraction, shape factor, and connectivity of the pores).

- The mathematical model is in qualitative agreement with the experimental results at $1225^{\circ} \mathrm{C}$ which shows a prolonged infiltration time for the feathery structure.

- A more realistic mathematical model considering the shape of the capillary tube as 'pipes with kernel inside' is proposed which reflects the real microstructure better, and hence predictions are closer to the measured infiltration depths.

\section{Acknowledgment}

The authors would like to express their gratitude to D. Peters for manufacturing the EB-PVD coatings.

\section{References}

[1] W. Braue, P. Mechnich, D.J. Green, 'Recession of an EB-PVDYSZ coated turbine blade by $\mathrm{CaSO}_{4}$ and Fe, Ti-rich CMAS-type deposits, J. Am. Ceram. Soc. 94 (12) (2011) 4483-4489.

[2] A.R. Krause, H.F. Garces, G. Dwivedi, A.L. Ortiz, S. Sampath, N.P. Padture, 'Calcia-magnesia-alumino-silicate (CMAS)-induced degradation and failure of air plasma sprayed yttria-stabilized zirconia thermal barrier coatings', Acta Mater. 105 (2016) 355-366.

[3] A.R. Krause, B.S. Senturk, H.F. Garces, G. Dwivedi, A.L. Ortiz, S. Sampath, N.P. Padture, D.J. Green, $2 \mathrm{ZrO}_{2} \cdot \mathrm{Y}_{2} \mathrm{O}_{3}$ Thermal barrier coatings resistant to degradation by molten CMAS: part I, optical basicity considerations and processing', J. Am. Ceram. Soc. 97 (12) (2014) 3943-3949.

[4] P. Mechnich, W. Braue, J. Smialek, 'Solid-State CMAS corrosion of an EB-PVD YSZ coated turbine blade: $\mathrm{Zr}^{4+}$ partitioning and phase evolution', J. Am. Ceram. Soc. 98 (1) (2015) 296-302.

[5] B. Mohan, T. Yuan, V. Desai, Y.H. Sohn, 'Degradation of yttria stabilized zirconia thermal barrier coatings by molten CMAS ( $\left.\mathrm{CaO}-\mathrm{MgO}-\mathrm{Al}_{2} \mathrm{O}_{3}-\mathrm{SiO}_{2}\right)$ deposits, Mater. Sci. Forum 595-598 (2008) 207-212.

[6] R. Naraparaju, U. Schulz, P. Mechnich, P. Döbber, F. Seidel, 'Degradation study of $7 \mathrm{wt}$ \% yttria stabilised zirconia (7YSZ) thermal barrier coatings on aero-engine combustion chamber parts due to infiltration by different CaO-MgO-Al2O3-SiO2 variants', Surf. Coat. Technol. 260 (2014) 73-81.

[7] J.L. Smialek, F.A. Archer, R.G. Garlick, 'The chemistry of Saudi-Arabian sand - a deposition problem on helicopter turbine airfoils', Adv. Mater.: Meeting Econ. Challenge/Adv. Synth. Processes 24 (1992) M63-M77.

[8] M.P. Borom, C.A. Johnson, L.A. Peluso, 'Role of environmental deposits and operating surface temperature in spallation of air plasma sprayed thermal barrier coatings, Surf. Coatings Technol. 86 (1-3)(1996) 116-126. 
[9] C.G. Levi, J.W. Hutchinson, M.H. Vidal-Setif, C.A. Johnson, 'Environmental degradation of thermal-barrier coatings by molten deposits', MRS Bull. 37 (10) (2012) 932-941.

[10] V.L. Wiesner, N.P. Bansal, 'Mechanical and thermal properties of calcium-magnesium aluminosilicate (CMAS) glass, J. Eur. Ceram. Soc. 35 (10) (2015) 2907-2914.

[11] C. Mercer, S. Faulhaber, A.G. Evans, R. Darolia, 'A delamination mechanism for thermal barrier coatings subject to calcium-magnesium-alumino-silicate (CMAS) infiltration', Acta Mater. 53 (4) (2005) 1029-1039.

[12] S. Krämer, J. Yang, C.G. Levi, 'Infiltration-inhibiting reaction of gadolinium zirconate thermal barrier coatings with CMAS melts', J. Am. Ceram. Soc. 91 (2) (2008) 576-583.

[13] J.M. Drexler, C.-H. Chen, A.D. Gledhill, K. Shinoda, S. Sampath, N.P. Padture, 'Plasma sprayed gadolinium zirconate thermal barrier coatings that are resistant to damage by molten $\mathrm{Ca}-\mathrm{Mg}-\mathrm{Al}-$ silicate glass, Surf. Coat. Technol. $206(19-20)(2012) 3911-3916$.

[14] P. Mechnich, W. Braue, 'Volcanic Ash-induced decomposition of EB-PVD $\mathrm{Gd}_{2} \mathrm{Zr}_{2} \mathrm{O}_{7}$ thermal barrier coatings to Gd-oxyapatite, zircon, and $\mathrm{Gd}$ Fe-Zirconolite', J. Am. Ceram. Soc. 96 (6) (2013) 1958-1965.

[15] A. Aygun, A.L. Vasiliev, N.P. Padture, X. Ma, 'Novel thermal barrier coatings that are resistant to high-temperature attack by glassy deposits', Acta Mater. 55 (20) (2007) 6734-6745.

[16] N.K. Eils, P. Mechnich, W. Braue, 'Effect of CMAS deposits on MOCVD coatings in the system $\mathrm{Y}_{2} \mathrm{O}_{3}-\mathrm{ZrO}_{2}$ : phase relationships', J. Am. Ceram. Soc. 96 (10) (2013) 3333-3340.

[17] A. F. Renteria, A small-angle scattering analysis of the influence of manufacture and thermal induced morphological changes on the thermal conductivity of EB-PVD PYSZ Thermal Barrier Coatings. Aachen, RWTH, 2006. [thesis].
[18] A.F. Renteria, B. Saruhan, U. Schulz, H.J. Raetzer-Scheibe, J. Haug, A. Wiedemann, 'Effect of morphology on thermal conductivity of EB-PVD PYSZ TBCs', Surf. Coatings Technol. 201 (6) (2006) 2611-2620.

[19] P. Naraparaju, U. Schulz, G.C. Mondragon Rodriguez, The accelerating effect of $\mathrm{CaSO}_{4}$ within CMAS ( $\left.\mathrm{CaO}-\mathrm{MgO}-\mathrm{Al}_{2} \mathrm{O}_{3}-\mathrm{SiO}_{2}\right)$ and its effect on the infiltration behavior in EB-PVD 7YSZ, J. Am. Ceram. Soc. (2016), n/a-n/a.

[20] D.B. Ingham, I. Pop, Transport Phenomena in Porous Media II, Elsevier sciene Ltd, Amsterdam, 2002.

[21] P.C. Carman, 'Fluid flow through granular beds', Chem. Eng. Res. Design 75 (1997) S32-S48.

[22] R.P. Chapuis, M. Aubertin, 'On the use of the Kozeny-Carman equation to predict the hydraulic conductivity of soils', Can. Geotech. J. 40 (3) (2003) 616-628.

[23] L. Pisani, 'Simple expression for the tortuosity of porous media', Transp. Porous Media 88 (2) (2011) 193-203.

[24] P.C. Carman, Flow of Gases Through Porous Media, Butterworths ScientificPublications, London, 1956

[25] K. Yazdchi, S. Srivastava, S. Luding, 'Microstructural effects on the permeability of periodic fibrous porous media', Int. J. Multiphase Flow 37 (8) (2011) 956-966.

[26] H.B. Zhao, C.G. Levi, H.N.G. Wadley, 'Molten silicate interactions with thermal barrier coatings', Surf. Coatings Technol. 251 (2014) 74-86.

[27] V.L. Wiesner, N.P. Bansal, 'Mechanical and thermal properties of calcium-magnesium aluminosilicate (CMAS) glass', J. Eur. Ceram. Soc. 35 (10) (2015) 2907-2914.

[28] J. Dvorkin, 'Kozeny-Carmen Equation Revisited', 2009, pangea.stanford.edu/ jack/KC_2009_JD.pdf. 